\title{
Nanoscale Characterization of Materials for Magnetic Recording Applications
}

\author{
David J. Larson
}

Imago Scientific Instruments Corp., 6300 Enterprise Lane, Madison, WI 53719-1193 USA.

In order to accelerate learning and shorten research and development cycle lifetimes in devices based on nanoscale magnetic materials [1], it is important to correlate processing conditions and physical properties with microstructural characterization. The atom probe combines quantitative 3-D atomicscale imaging with high analytical sensitivity [2-4]. This technique, known as atom probe tomography [3], operates by taking needle-shaped specimens apart one atom at a time. Individual specimen atoms are ionized from the surface of a sharp specimen by a rapidly-pulsed electric field, and are then accelerated to a position-sensitive detector. The location where each ionized atom impacts this detector directly maps to its original specimen position by projection microscopy, while time-of-flight measurement determines elemental identity.

Magnetoresistive spin valves and tunnel junctions are two structures commonly used to form the "reader" portion of magnetic recording heads. Fig. 1 shows atom maps of a simple tunnel junction containing an alumina barrier. A composition profile across the barrier is shown in Fig. 2 [5,6] where the composition of the barrier region is determined to be $\sim\left(\mathrm{Al}_{2} \mathrm{O}_{3}\right)_{80} \mathrm{Co}_{20}$. In addition, significant diffusion of oxygen into the lower Co layer is detected in this structure.

Spin valve structures use thin alternating layers of $\mathrm{Co}$ and $\mathrm{Cu}$ to produce an electron spin-dependent resistance change as the reader moves across individual bits in magnetic recording media. Interface width values from composition profiles such as that shown in Fig. 3 (alternating $\mathrm{Co} / \mathrm{Cu}$ layers $\sim 1 \mathrm{~nm}$ thick) show that there is more undesired chemical intermixing of atoms when Co is deposited onto $\mathrm{Cu}$ than when $\mathrm{Cu}$ is deposited onto $\mathrm{Co}$ [7]. Another type of spin valve uses a "current confined path" containing a nano-oxide-layer with small metallic paths in order to increase the magnetoresistance while maintaining low overall device resistance. Fig. 4 shows the analysis of such a structure using the local electrode atom probe (LEAP) [4]. Fig. 4b shows quantification of the spatial distributions of alumina and $\mathrm{Cu}$ regions contained in the device using isoconcentration surfaces $[6,8]$.

Perpendicular recording media using oxygen to magnetically isolate individual grains in Co alloys [9] is currently being used to increase the recording density in hard drives. An oriented non-magnetic layer $(\mathrm{Ru})$ is used to seed the magnetic layer (Co alloy), Fig. 5, in order to engineer both grain size and crystallography. Fig 5a shows a cross section LEAP atom map (Co and $\mathrm{O}$ atom positions shown as light and dark dots, respectively) while Fig. 5b shows the plan view section (from the dashed box region in Fig 5a) where both $\mathrm{Si}$ and $\mathrm{O}$ segregation to the grain boundaries is observed [8].

[1] R. L. Comstock, J. Mat. Sci. Mat. Elec. 13(9) (2002) 509.

[2] A. Cerezo et al., Rev. Sci. Instrum. 69 (1998) 49.

[3] M. K. Miller, Atom Probe Tomography, Kluwar Academic Publishers, New York, 2000.

[4] T. F. Kelly et al., Micro. Microanal. 10(3) (2004) 373.

[5] A. K. Petford-Long et al., J. Appl. Phys. 98 (2005)124904.

[6] D. J. Larson, Thin Solid Films, In press.

[7] D. J. Larson et al., Acta Mater. 52(10) (2004) 2847.

[8] H. Fukuzawa et al., Digests of the IEEE International Magnetics Conference, (2005) p. GQ-03

[9] J. D. Risner et al, Micro. Microanal. 11(S2) (2005) 1806.

[10] The assistance of Drs. A. Cerezo and A. Petford-Long (Oxford University), Dr. H. Fukuzawa (Toshiba) and the many Imago employees who enabled this work is gratefully acknowledged. 

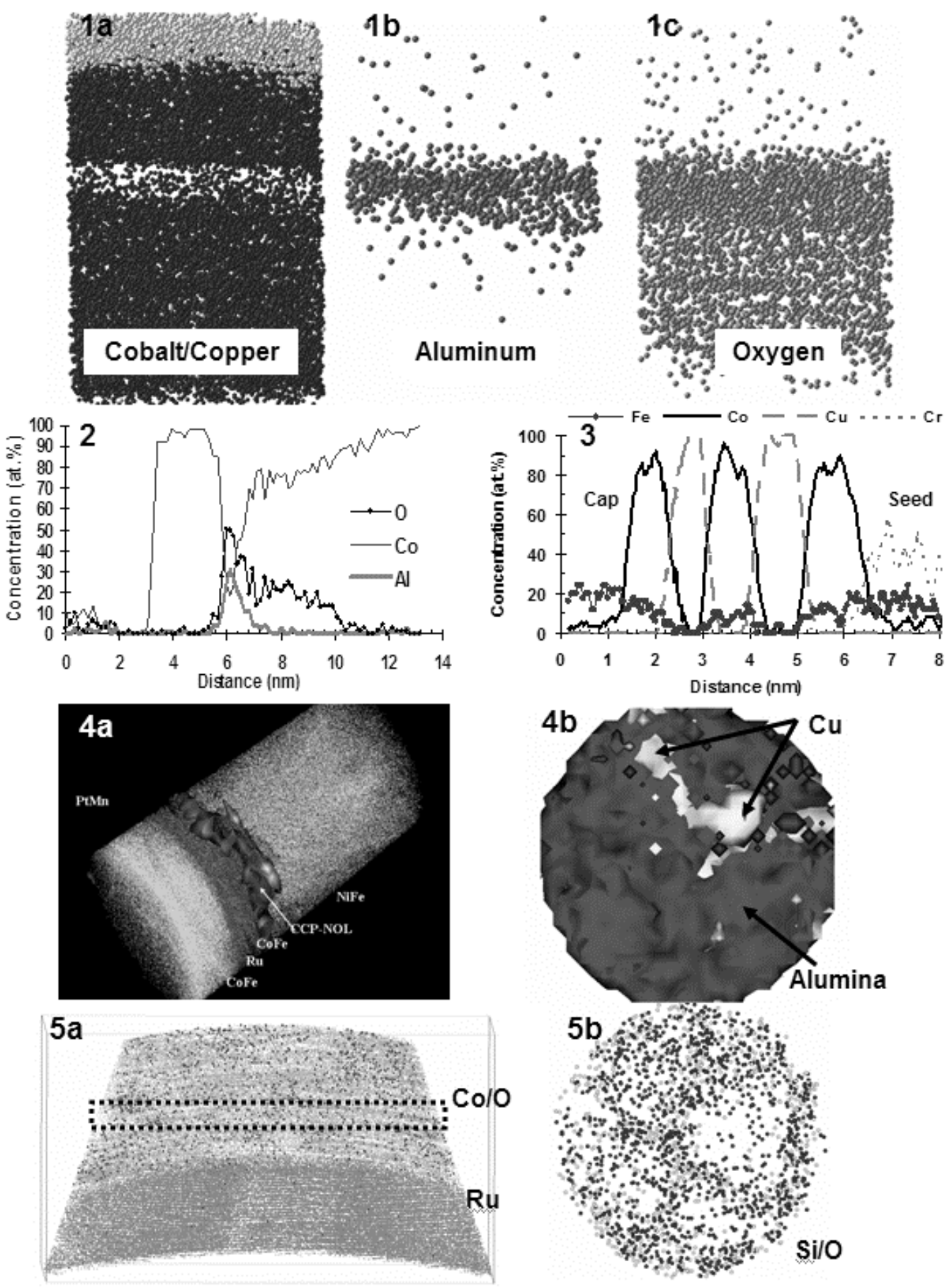

Fig. 1. Atom maps ( $\sim 8 \mathrm{~nm}$ by $12 \mathrm{~nm})$ of a) $\mathrm{Co}, \mathrm{b}) \mathrm{Al}$ and c) $\mathrm{O}$ in a simple tunnel barrier structure.

Fig. 2. Concentration profile normal to the barrier plane from the data shown in Fig. 1.

Fig. 3. Concentration profile across $\mathrm{Co}$ and $\mathrm{Cu}$ layers typically used in a "spin valve" structure.

Fig 4. a) Atom map ( $\sim 25 \mathrm{~nm}$ by $40 \mathrm{~nm})$ of a current confined path spin valve and b) isoconcentration surfaces ( $\sim 30 \mathrm{~nm}$ diameter) for the alumina (dark) and copper (light) regions contained in the spin valve.

Fig 5. a) Cross section atom map ( $\sim 20 \mathrm{~nm}$ by $35 \mathrm{~nm})$ and b) plan view section of Si (dark) and O (light) atoms showing grain boundary segregation. 